\title{
Attitude Stabilization of a Quad-Rotor UAV Using a Two-camera Vision System
}

\author{
Dae-Yeon Won* and Min-Jea Tahk** \\ Division of Aerospace Engineering, \\ School of Mechanical, Aerospace and Systems Engineering \\ Korea Advanced Institute of Science and Technology (KAIST), Daejeon, Korea 305-701
}

\begin{abstract}
This paper is mainly concerned with the vision-based attitude stabilization of a quad-rotor UAV. The methods for attitude control rely on computing the roll and pitch angles of the vehicle from a two-camera vision system. One camera is attached to the body-fixed $\mathrm{x}$-axis and the other to the body-fixed $\mathrm{y}^{-}$-axis. The attitude computation for the quad-rotor UAV is performed by image processing consisting of Canny edge and Hough line detection. A proportional and integral controller is employed for the attitude hold autopilot. In this paper, the quad-rotor UAV is modeled by 6 -DOF nonlinear equations of motion that includes rotor aerodynamics with blade element theory. The performance of the proposed method is evaluated through 3D environmental numerical simulations.
\end{abstract}

Key Word : Quad-Rotor UAV, Attitude stabilization, Vision-based control

\section{Introduction}

There has been an increased emphasis on the use of UAVs (unmanned aerial vehicles) in various activities and for both civilian and military applications. Such applications include surveillance, reconnaissance, target tracking, data acquisition, jamming, communications relay, decoy, harassment, ordnance delivery, or supply flights. Recent improvements in sensor technology, data processing, and actuator systems make possible the development of small UAVs under $30 \mathrm{~kg}$. For small UAVs, a quad-rotor UAV is an emerging rotorcraft concept. The quad-rotor UAV propelled by four rotors with two counter-rotating blades, has been developed to cover various types of unmanned missions, like small-area monitoring, building exploration, surveillance, and rescue.

The flight control system design of the quad-rotor UAV requires further investigation in the following areas: automatic control, machine vision, computer science, and sensor technology. Autonomous flight control system design challenges include robust controls, 3-D trajectory planning, multi-agent control and collision avoidance. Since the quad-rotor UAV is dynamically unstable, control algorithms are required for stabilization [1].

This paper presents an approach that combines vision-based attitude computation with an attitude hold autopilot system to stabilize the quad-rotor UAV. The motivation for the vision-based attitude computation is to design robust attitude stabilization for the quad-rotor UAV that uses two on-board cameras. The vision system is available in indoor and outdoor environment and one of the passive sensors. In addition, the vision system offers reasonable solution to reducing the payload and decreasing the manufacturing cost. An inertial navigation system that includes GPS and gyro needs more payload and has a high cost.

* Ph. D. Student

** Professor

E-mail : mitahk@fdcl.kaist.ac.kr

Tel : +82-42-869-3718

Fax : $+82-42-869-3710$ 
Because of the advantages it offers, previous research has applied a vision-based control technique to the quad-rotor UAV in various ways. A vision-based stabilization method with on-board and ground cameras was investigated by Altug et al [2]. Two cameras were set to see each other to estimate the full 6-DOF pose of the quad-rotor. At MIT, the vision-based estimation and control of the quad-rotor UAV using a single camera relative to the moire pattern target was undertaken by Tournier et al [3]. The on-board camera was mounted on the vehicle with the target in the field of view. This algorithm processes the image, and extracts the attitude and position information by utilizing geometry and four single-point discrete Fourier. Fowers et al proposed the vision-aided stabilization of the quad-rotor UAV. The vision-aided drift stabilization was achieved by means of Harris feature detection and template matching.

This paper is organized as follows: in the first section, the quad-rotor UAV dynamic model is presented. The second section proposed the vision-based stabilization strategy. In the third section illustrates the attitude hold autopilot design. The fourth section deals with numerical simulations. The final section summarizes the overall discussion.

\section{Quad-Rotor UAV Dynamic Model}

\section{Nonlinear Model}

The configuration of the quad-rotor UAV is a helicopter type configuration. The quad-rotor UAV provides lift force and thrust by a fuselage with four rotors. These rotors are mounted four ends of fuselage structure without pitch control components. Also, four rotors compensate the torque reaction of the each rotor to accomplish the balancing force and moment acting on the vehicle. The quad-rotor configuration uses four contra-rotating rotors so that the torques of the rotors are equal and opposing. Rotor 1 and rotor 3 rotate clockwise while rotor 2 and rotor 4 rotate counterclockwise. This configuration automatically balances the rotor torque in hovering without requiring tail rotor or power-absorbing auxiliary rotor.

The development of the 6-DOF nonlinear equations of motion for the quad-rotor UAV follows a similar way of the helicopter type UAV. The equations of motion for a rigid-body are derived based on the nonlinear aircraft and miniature rotorcraft models [4], [5]. Since the quad-rotor UAV is a vehicle that is free to simultaneously rotate and translate in 6-DOF, the rigid-body dynamics are derived by the Newton's laws [4]. Fig. 1 shows the quad-rotor UAV with the conventional right-handed set of body-fixed axes and inertial axes. For the quad-rotor $\mathrm{UAV}$, the 6-DOF rigid-body equations of motions are expressed the differential equations describing the translational motion, rotational motion and kinematics.

$$
\dot{\boldsymbol{\eta}}=\mathbf{R}(\boldsymbol{\eta}) \boldsymbol{\omega}
$$

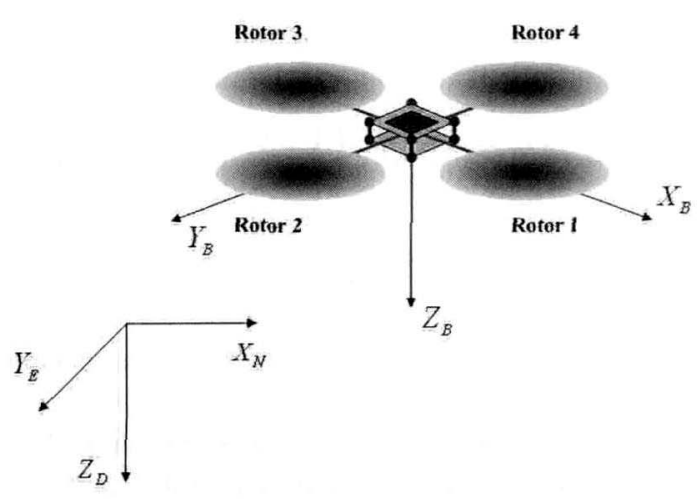

Fig. 1. Quad-Rotor UAV Configuration 


$$
\begin{gathered}
m \dot{\mathbf{v}}_{B}+\boldsymbol{\omega} \times m \mathbf{v}_{B}=\mathbf{F}_{B}+m \mathbf{g} \\
\mathbf{I} \dot{\boldsymbol{\omega}}=-\boldsymbol{\omega} \times \mathbf{I} \boldsymbol{\omega}+\mathbf{u}+\mathbf{u}_{g}
\end{gathered}
$$

where $\mathbf{v}_{B}=\left[\begin{array}{lll}u & v & w\end{array}\right]^{\top}$ is the vector of longitudinal, lateral and vertical body velocities, $\boldsymbol{\eta}=\left[\begin{array}{lll}\phi & \theta & \psi\end{array}\right]^{T}$ is the vector of roll, pitch and yaw angles, $\boldsymbol{\omega}=\left[\begin{array}{lll}P & Q & R\end{array}\right]^{T}$ is the vector to describe roll, pitch and yaw body rates and $\mathbf{I}=\operatorname{diag}\left[I_{x}, I_{y}, I_{z}\right]$ is the inertia matrix. The transition matrix of the body-fixed frame with respect to the inertial frame by means of Euler angles.

$$
\mathbf{R}=\left[\begin{array}{ccc}
\cos \psi \cos \theta & \sin \psi \cos \theta & -\sin \theta \\
-\sin \psi \cos \phi+\cos \psi \sin \theta \sin \phi & \cos \psi \cos \phi+\sin \psi \sin \theta \sin \phi & \cos \theta \sin \phi \\
\sin \psi \sin \phi+\cos \psi \sin \theta \cos \phi & -\cos \psi \sin \phi+\sin \psi \sin \theta \cos \phi & \cos \theta \cos \phi
\end{array}\right]
$$

$\mathbf{F}_{B}$ is the vector used to describe external force components with respect to the body-fixed frame. The external force components can be written as Eq. (5).

$$
\mathbf{F}_{B}=\left[\begin{array}{c}
0 \\
0 \\
\sum T_{i}
\end{array}\right], \quad \mathbf{g}=\left[\begin{array}{c}
-g \sin \theta \\
g \cos \theta \sin \phi \\
g \cos \theta \cos \phi
\end{array}\right]
$$

Total external control moments $\mathbf{u}$ and gyroscopic moments $\mathbf{u}_{\mathrm{g}}$ acting on the quad-rotor UAV induced by rotor thrust and gyroscopic effect can be written as Eq. (6). Different position and rotating direction leads different control effectiveness of each rotor.

$$
\mathbf{u}=\left[\begin{array}{c}
d\left(T_{4}-T_{2}\right) \\
d\left(T_{1}-T_{3}\right) \\
\sum Q_{i}
\end{array}\right], \quad \mathbf{u}_{g}=\left[\begin{array}{c}
I_{r} Q(-1)^{i+1} \Omega_{i} \\
I_{r} P(-1)^{i} \Omega_{i} \\
0
\end{array}\right], i=1,2,3,4
$$

where $T_{i}$ is the thrust induced by rotor i and $Q_{i}$ is the torque induced by rotor i. $I_{r}$ is the inertia of the rotor and $d$ is the distance between center of gravity and center of rotor. Rotor thrust and torque produced by each rotor are computed by using Eqs. (7)-(10) based on the blade element theory. The blade element theory forms the basis of most modern analysis of rotor aerodynamics because it provides overall rotor performance in terms of blade pitch angle, twist, and airfoil shape [6], [7]. Thrust coefficient $C_{T}$ and torque coefficient $C_{Q}$ are given by Eq. (9) and Eq. (10).

$$
\begin{gathered}
T_{i}=(0.5) \rho\left(\Omega_{i} R\right)^{2} \pi R^{2}\left(C_{T}\right)_{i} \\
Q_{i}=(0.5) \rho\left(\Omega_{i} R\right)^{2} \pi R^{3}\left(C_{Q}\right)_{i} \\
C_{T}=\frac{a \sigma}{2}\left[\left(\frac{2}{3}+\mu_{x}^{2}\right) \theta_{0}+\left(\frac{1}{2}+\frac{\mu_{x}^{2}}{2}\right) \theta_{1}-\left(\mu_{z}+\lambda_{i}\right)\right] \\
C_{Q}=\frac{C_{D_{0}} \sigma}{4}\left(1+3 \mu_{x}^{2}\right)+\left(\mu_{z}+\lambda_{i}\right) C_{T}
\end{gathered}
$$

where $a$ is two dimensional lift-curve-slope of the airfoil section comprising the rotor, $\theta_{0}$ is the blade collective pitch angle, $\theta_{1}$ is the blade twist angle, $b$ is the number of blade, $c$ is the chord length, $\sigma$ is the rotor solidity ratio, $\Omega$ is the rotor speed, $R$ is the rotor radius, $\rho$ is the air density, and $v_{i}$ is the induced velocity. To evaluate $C_{T}$ and $C_{Q}$, it is necessary to predict the induced velocity ratio. Then it is possible to find closed form solutions for $C_{T}$ and $C_{Q}$ in terms of the operational and geometric parameters of the rotor. 


\section{Control allocation}

Since the quad-rotor UAV has four rotors and uses only angular velocity control to make motion, the actual control commands should be allocated as the angular velocity for each rotor in the control system. There can be inconsistence in the control system structure and unexpected performance according to the control allocation. Therefore, we have to choose a proper way to allocate control commands. Thus the conventional method of the helicopter control system is adopted for the control allocation. Generally, as helicopter control command, four control channels altitude, pitch, roll, and yaw are needed. These commands are expressed as $\delta_{\mathrm{COL}}, \delta_{\mathrm{LON}}, \delta_{\mathrm{LAT}}$, and $\delta_{\text {DIR, }}$ respectively. Each rotor contributes to the three channels for the altitude, yaw, pitch, or roll. Eq. (11) describes control allocation method applied to the quad-rotor UAV.

$$
\begin{aligned}
& \Omega_{1}=\Omega_{n o m}+\left(\delta_{C O L} / 4\right)+\left(\delta_{L O N} / 2\right)-\left(\delta_{D I R} / 4\right) \\
& \Omega_{2}=\Omega_{n o m}+\left(\delta_{C O L} / 4\right)-\left(\delta_{L A T} / 2\right)+\left(\delta_{D I R} / 4\right) \\
& \Omega_{3}=\Omega_{n o m}+\left(\delta_{C O L} / 4\right)-\left(\delta_{L O N} / 2\right)-\left(\delta_{D I R} / 4\right) \\
& \Omega_{4}=\Omega_{n o m}+\left(\delta_{C O L} / 4\right)+\left(\delta_{L A T} / 2\right)+\left(\delta_{D I R} / 4\right)
\end{aligned}
$$

\section{Vision-based Attitude Stabilization}

The basic idea of the method proposed in this section is to compute the roll and pitch angles from two on-board cameras attached to the body-fixed $\mathrm{x}$-axis and $\mathrm{y}$-axis, respectively. The attitude angles are induced from angles between the vertical axis of the camera coordinate frame and vertical lines detected in the real world. Because the vertical lines can be easily obtained through partition, ceiling and floor, the proposed method provides enough information to stabilize the vehicle. The first part of this section focuses on the attitude stabilization strategy. In the second section, the Canny edge detection and Hough line detection algorithms are introduced; these permit vertical lines to be detected from the image. Numerical simulations have been carried out to assess the performance of the proposed method.

\section{Attitude Stabilization Strategy}

The attitude stabilization strategy is composed of three main steps: obtaining the image, detecting the edges in the image, and computing the roll and pitch angles. The computed roll and pitch angles are used by attitude hold autopilot system. Edge detection is performed by the Canny edge detector, line detection through the Hough line detector. The approach taken here proposes to use, the Hough line detection for the roll and pitch angle computation, in order to obtain a fast algorithm. With that aim in mind, the approach assumes that vertical lines are in the field of view of two on-board cameras and that altitude is controlled separately. Since the vertical lines can be easily detected from buildings, ceilings and partitions and since altitude information can be obtained from other sensor like sonar, these assumptions are reasonable. On that basis, the proposed method is effective because it does not need other specific targets. Fig. 2. illustrates the vision-based attitude stabilization strategy.

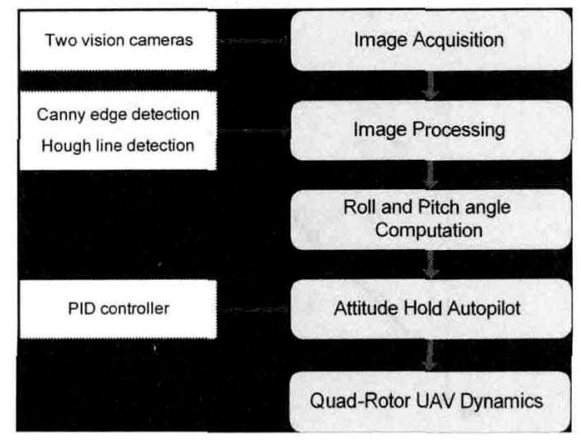

Fig. 2. Vision-based attitude stabilization strategy 


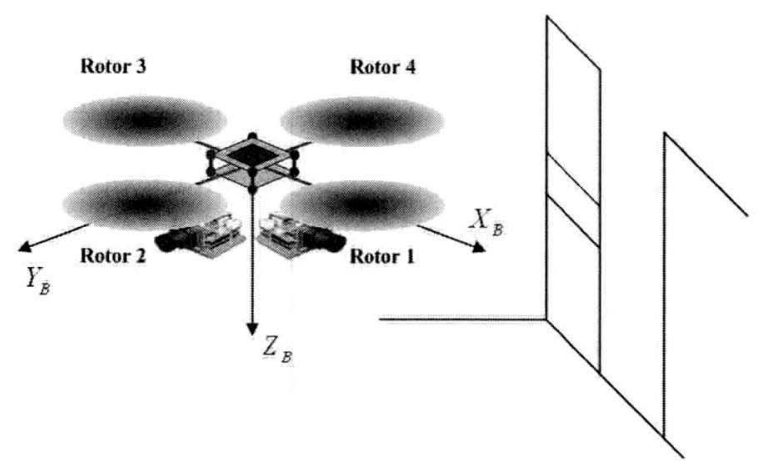

Fig. 3. Two-Vision System for attitude stabilization

The two-camera attitude computation method involves the use of two on-board cameras that are attached along the body-fixed $\mathrm{x}$-axis and the body-fixed $\mathrm{y}$-axis, respectively. The configuration of the camera system is shown in Fig. 3. The camera attached along the body-fixed $\mathrm{x}$-axis is used to compute the roll angle; the camera attached along the body-fixed $\mathrm{y}$-axis is used for the pitch angle. Although the computed roll and pitch angles do not exactly match with the true values, the error values converge to zero while the roll and pitch angles close to zero.

\section{Canny Edge Detection}

The Canny edge detection is used to detect edges in images. The Canny edge detection operator is introduced by John F. Canny in 1986 and developed as a popular algorithm to detect edges. It uses a multi-state algorithm to detect a wide range of edges in images. Canny used the calculus of variations to discover the optimal edge detection algorithm so that edge detection satisfies good detection and localization. The optimal function in Canny edge detection is described by the sum of four exponential terms, but these terms can be approximated by the first derivative of the Gaussian. The Canny edge detection algorithm contains two parameters; the size of the Gaussian filter and threshold. These parameters adjustable for the image quality affect the computational time and effectiveness of the algorithm. Detailed derivation and computational method for the Canny edge detection are introduced in [8]. Binary images in which each pixel is 1 if an edge pixel, 0 otherwise are obtained after performing edge detection.

\section{Hough Line Detection}

The Hough transform is used to detect lines in binary image obtained from the Canny edge detection. The key idea of the Hough transform is to map lines into a simple peak detection problem in the space of the parameters of the curve. To transform line intersection in a simple peak problem, the polar representation for the line defined by Eq. (12) is used.

$$
\eta=x \cos \xi+y \sin \xi
$$

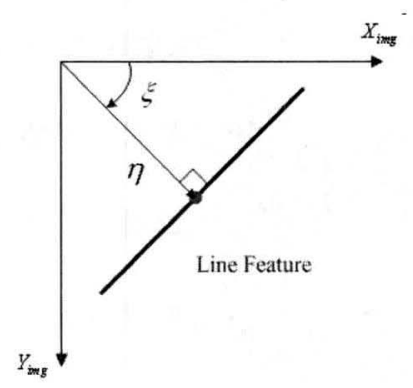

Fig. 4. Polar representation parameters 
where $n$ is the distance between the image origin and the line and $\xi$ is the line orientation. A point on the $x^{-} \mathrm{y}$ coordinate image plane is represented by a sinusoid, not a line, in transformed parameter space. The intervals of variation of $n$ and $\xi$ are finite, and any line can be represented. The specific Hough line detection algorithm is described as follows [8].

i. The input is $E$, a $M \times N$ binary image in which each pixel $E(i, j)$ is 1 if an edge pixel, 0 otherwise. Let $\eta_{d}$ and $\xi_{d}$ be the arrays containing the discretized intervals of the $\pi$ and $\xi$ parameter spaces, and $K, P$, respectively, their number of elements.

ii. Discretize the parameter spaces of $\eta$ and $\xi$ using sampling $\delta_{n}, \delta_{\xi}$, which must yield acceptable resolution and manageable size for $n_{d}$ and $\xi_{d}$.

iii. Let $A(K, P)$ be an array of integer counters; initialize all elements of $A$ to zero.

iv. For each pixel, such that $E(i, j)=1$ and for $p=1 \ldots P$.

(a) Let $n=i \cos \xi_{d}(p)+j \sin \xi_{d}(p)$;

(b) Find the index, $k$, of the element of $n_{d}$ closest of $n$;

(c) Increment $A(K, P)$ by one.

v. Find all local maxima $(k, p)$ such that $A(k, p)>t_{h}$, where $t_{h}$ is user-defined threshold.

\section{Attitude Computation}

The attitude of the quad-rotor UAV is computed from the line information detected by Hough line detection. The length of line parameters longer than user-defined threshold is used to measure the attitude of the vehicle. The roll and pitch angles are computed by taking the mean value of detected line angle parameters by the image processing. This attitude computation method is available under the following assumptions.

1) More than one vertical line is exist in field of view.

2) There is no non-horizontal and vertical lines longer than user-defined threshold.

3) Range of true roll and pitch angle is $\left[-40^{\circ},+40^{\circ}\right]$

Figs. 5 and 6 show the image processing results on the test image taken by the vision camera. In this case, 8 lines are detected and can be used to compute the attitude of the vehicle. Table 1 shows the parameters of detected 8 lines by the Hough line detection. If the test image taken by the camera attached the body-fixed $\mathrm{x}$-axis, the mean and standard deviation of the roll angle are $18.00^{\circ}$ and $1.51^{\circ}$, respectively.

Table 1. Detected Line Parameters

\begin{tabular}{|c|c|c|c|c|c|c|c|c|}
\hline line & $(1)$ & $(2)$ & $(3)$ & $(4)$ & $(5)$ & $(6)$ & $(7)$ & $(8)$ \\
\hline (pix) & -363 & -134 & -358 & -423 & 198 & -84 & 286 & -307 \\
\hline (deg) & 163 & 163 & 163 & 159 & 71 & 163 & 71 & 163 \\
\hline
\end{tabular}

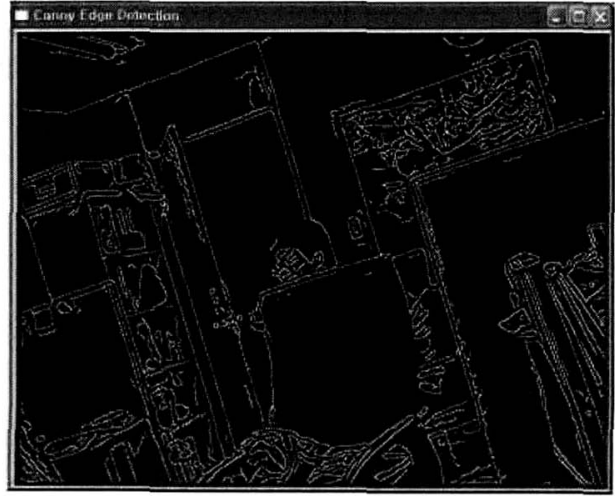

Fig. 5. Canny Edge Detection

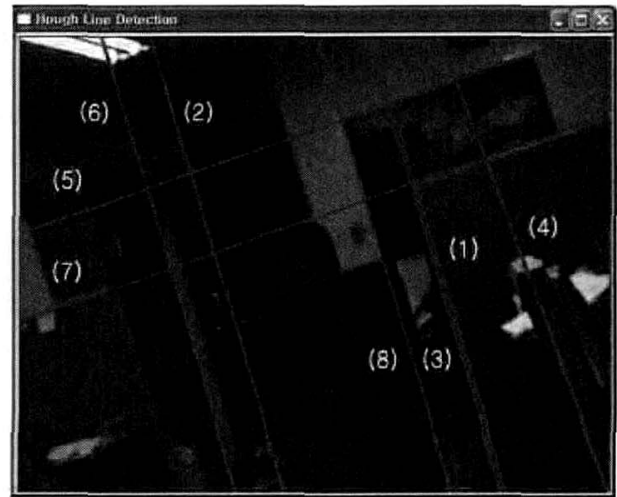

Fig. 6. Hough Line Detection 


\section{Attitude Hold Autopilot Design}

The control objective becomes to stabilize the roll and pitch angle, and hold them. The linear proportional integral controller in Fig. 7 is employed for the attitude hold autopilot. The attitude hold autopilot is consists of a double-loop which inner-loop is the angular rate feedback and outer-loop is the attitude feedback.

A phase-lead compensation and proportional integral controller are necessary for a small steady-state error and good transient response. Inner-loop rate feedback is used to provide short-period damping. Since the quad-rotor UAV is symmetrical with respect to the $X_{B}$, and $Y_{B}$ axes, gains for pitch and roll autopilots are identical. The classical proportional integral controller is designed with corresponding classical robustness measures of gain and phase margins. The controller for the attitude hold autopilot is defined as follows:

$$
G(s)=K_{P}\left(\frac{s+K_{I}}{s}\right)\left(\frac{s+K_{D}}{s+10 K_{D}}\right)
$$

\section{Numerical Simulation}

\section{Virtual Reality Toolbox}

To verified the proposed vision-based stabilization strategy, numerical simulation is performed. The virtual images from two-camera vision system of quad-rotor UAV made by using a Virtual Reality Toolbox. The Virtual Reality Toolbox supported by MATLAB/Simulink provides a solution for viewing and interacting with dynamic systems in a $3 \mathrm{D}$ virtual reality environment. It has the capabilities of the MATLAB/Simulink into the world of virtual reality graphics and gives a realistic simulation to be used as a test-bed for performance evaluation of the vision system [9], [10]. A number of adjustable parameters for the virtual environment and cameras allow for testing different setups. These parameters include zoom factor, field of view and pointing angles referenced from the vehicle.

\section{Simulation Results}

The simulation modeling for nonlinear quad-rotor UAV dynamics and environmental visualization is performed based on MATLAB/Simulink. Figs. 8-11 show the numerical simulation results in the attitude stabilization of the quad-rotor UAV by using the two-camera vision system. The vector for the initial Euler rotation angles is $\left[15^{\circ}, 10^{\circ}, 0^{\circ}\right]$. In this simulation scenario, we assume that the vertical line components are exists in the field of view of the vision system. Autopilot commands update and image processing are performed at $20 \mathrm{~Hz}$.

Since the proposed vision stabilization strategy cannot detect yaw angle, the vehicle drifts slightly during the flight. From the results shown in Fig 8, we observe that the vision-based autopilot stabilizes the quad-rotor UAV within 3 seconds and holds it. Fig. 9 illustrates the time histories of the four control inputs. The vision-based attitude autopilot stabilized the vehicle by the longitudinal and lateral inputs while the altitude is controlled by the collective input.

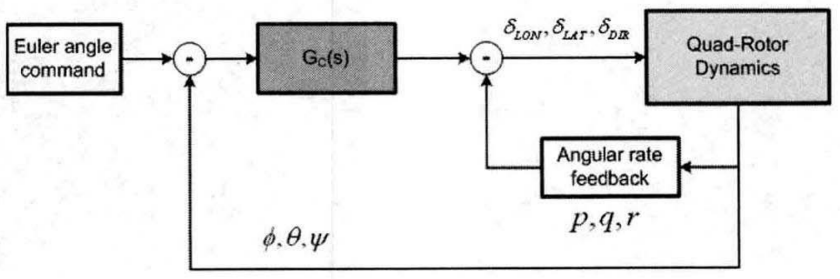

Fig. 7. The control architecture of Attitude hold autopilot 

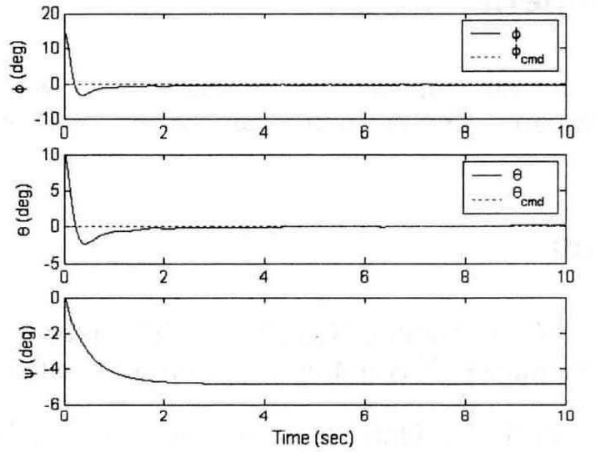

Fig. 8. Euler angles
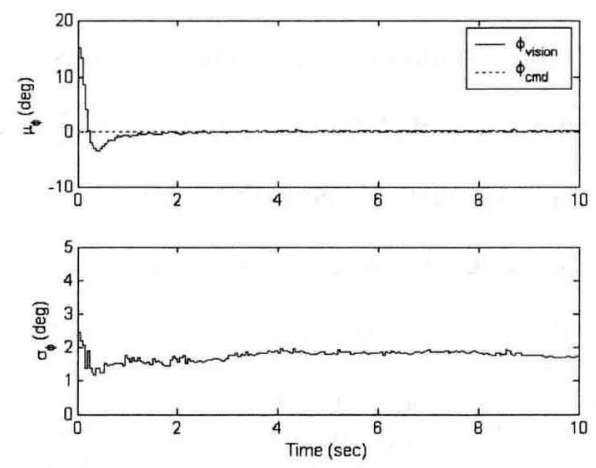

Fig. 10. Roll angle computation: mean and standard deviation

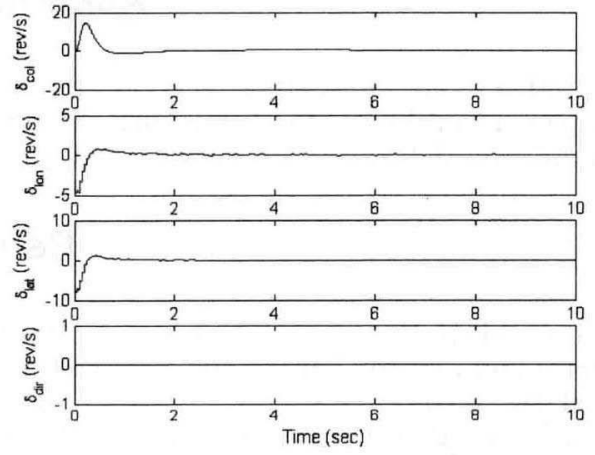

Fig. 9. Control inputs
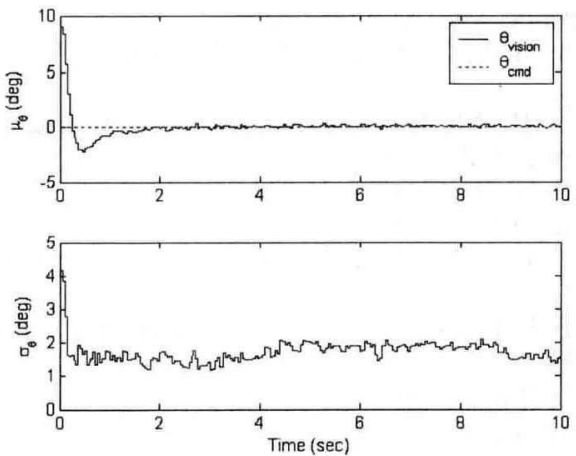

Fig. 11. Pitch angle computation: mean and standard deviation

Time histories of the roll and pitch angle computations are shown in Figs 10 and 11 . At the beginning, the standard deviations of the roll and pitch angles are more than $2^{\circ}$. However, the standard deviations are decreased by almost $1^{\circ}$, while the attitude is stabilized within $3^{\circ}$. Since the accuracy of attitude is affected by the motion of the vehicle, the accuracy of attitude angles are low at the beginning. With this method, the large roll angle makes the bias error in the pitch angle and the large pitch angle makes the bias error in the roll angle. Also, the small amount of variations in roll and pitch angle are caused by the vision system's truncation error. To improve the performance capability of the proposed method, the user-defined parameters of the image processing algorithm should be chosen appropriately, depending on the flight environments.

\section{Conclusions}

This paper is concerned with vision-based attitude stabilization technique. The proposed algorithm can be applied to a quad-rotor UAV operating in urban and indoor environments. Attitude information for the attitude hold autopilot computed from the image processing algorithm includes both Canny edge detection and Hough line detection. The simulation results of the quad-rotor UAV with a two-camera vision system demonstrate the effectiveness and performance with the proposed stabilization strategy in spite of its limitations. The findings from the proposed method suggest developing a low-cost and effective attitude stabilization system with two on-board cameras. Further work being undertaken includes a hardware implementation of the vision system. 


\section{Acknowledgement}

Authors are gratefully acknowledging the financial support by Agency for Defense Development and by UTRC(Unmanned Technology Research Center) and Brain Korea 21 Project, Korea Advanced Institute of Science and Technology.

\section{References}

1. G. M. Hoffmann, H. Huang, S. L. Waslander and C. J. Tomlin, "Quadrotor Helicopter Flight Dynamics and Control Theory and Experiment", AIAA Guidance, Navigation and Control Conference and Exhibit, 2007.

2. E. Altug, J. P. Ostrowski and C. J. Taylor, "Control of a Quadrotor Helicopter Using Dual Camera Visual Feedback", The International Journal of Robotics Research, Vol. 24, No. 5, pp. 329-341, 2005.

3. G. P. Tournier, M. Valenti, J P. How and E. Feron, "Estimation and Control of a Quadrotor Vehicle Using Monocular Vision and Moire Patterns", AIAA Guidance, Navigation, and Control Conference and Exhibit, 2006. 1992.

4. B. L. Stevens and F. L. Lewis, Aircraft control and simulation, John Wiley \& Sons, INC,

5. B. Mettler, Identification Modeling and Characteristics of Miniature Rotorcraft, Kluwer Academic Publisher, 2003.

6. R. W. Prouty, Helicopter Performance, Stability, and Control, Krieger Publishing Company, Malabar, FL, 1990. 2000.

7. J. G. Leishman, Principles of Helicopter Flight, Cambridge University Press, New York, NY,

8. E.Trucco and A.Verri, Introductory Techniques for 3-D Computer Vision, Prentice Hall, Chap $5.5,1998$.

9. The MathWorks, Inc., Virtual Reality Toolbox User's Guide, 2004.

10. Castillo-Effen, M, Alvis, W. and Castillo, C., 2005, "Modeling and visualization of multiple autonomous heterogeneous vehicles", IEEE International Conference on Systems, Man and Cybernetics, Vol. 3, pp. 2001-2007. 